Meta

Journal des traducteurs

Translators' Journal

\title{
Nom propre en russe : problèmes de traduction
}

\section{Sergueï Sakhno}

Volume 51, numéro 4, décembre 2006

La traduction des noms propres (1) et Langue, traduction et mondialisation : interactions d'hier, interactions d'aujourd'hui Language, Translation and Globalization: Interactions from Yesterday, Interactions from Today (2)

URI : https://id.erudit.org/iderudit/014336ar

DOI : https://doi.org/10.7202/014336ar

Aller au sommaire du numéro

Éditeur(s)

Les Presses de l'Université de Montréal

ISSN

0026-0452 (imprimé)

1492-1421 (numérique)

Découvrir la revue

Citer cet article

Sakhno, S. (2006). Nom propre en russe : problèmes de traduction. Meta, 51(4), 706-718. https://doi.org/10.7202/014336ar

\section{Résumé de l'article}

En dehors d'exemples bien connus (mais insuffisamment étudiés d'un point de vue proprement linguistique) de transcriptions quasi phonétiques (Popoff) et de translittérations (Popov), les pratiques existantes pour rendre les noms propres russes en français présentent plusieurs cas intermédiaires qui n’ont pas été systématisés ni décrits dans des termes linguistiques stricts. Nous proposons une classification de travail basée sur des données trouvées sur des sites web français concernant les variations des graphies de certains noms tels que Gorbatchev, Eltsine, Ekaterinbourg.

Parmi d'autres questions abordées, notons les difficultés liées au système anthroponymique russe (patronymes, diminutifs, traduction de noms " parlants ", connotés, dans des textes littéraires) et celles qui sont la conséquence des évolutions politiques récentes dans l'espace post-soviétique (débaptisations, rebaptisations, autochtonisation toponymiques).
Ce document est protégé par la loi sur le droit d'auteur. L’utilisation des services d’Érudit (y compris la reproduction) est assujettie à sa politique d'utilisation que vous pouvez consulter en ligne.

https://apropos.erudit.org/fr/usagers/politique-dutilisation/ 


\title{
Nom propre en russe: problèmes de traduction
}

\author{
SERGUEÏ SAKHNO \\ Université Paris 10, Paris, France \\ ssakhno@u-paris10.fr
}

\begin{abstract}
RÉSUMÉ
En dehors d'exemples bien connus (mais insuffisamment étudiés d'un point de vue proprement linguistique) de transcriptions quasi phonétiques (Popoff) et de translittérations (Popov), les pratiques existantes pour rendre les noms propres russes en français présentent plusieurs cas intermédiaires qui n'ont pas été systématisés ni décrits dans des termes linguistiques stricts. Nous proposons une classification de travail basée sur des données trouvées sur des sites web français concernant les variations des graphies de certains noms tels que Gorbatchev, Eltsine, Ekaterinbourg.

Parmi d'autres questions abordées, notons les difficultés liées au système anthroponymique russe (patronymes, diminutifs, traduction de noms «parlants», connotés, dans des textes littéraires) et celles qui sont la conséquence des évolutions politiques récentes dans l'espace post-soviétique (débaptisations, rebaptisations, autochtonisation toponymiques).
\end{abstract}

\begin{abstract}
Beyond well known (yet insufficiently questioned from a linguistic point of view) samples of quasi-phonetic transcription (Popoff) and translitteration (Popov), the existing practice of rendering Russian proper names into French displays many intermediate cases which have been neither systematized nor described in strict linguistic terms. We propose a working classification based on data of French web sites concerning such names as Gorbachev, Eltsin, Ekaterinbourg.

Among other problems discussed in the paper: difficulties linked to Russian anthroponomical system (patronymics, diminutives, possibility of translating connotated "meaningful" names in literary texts) and those involved by recent political events in the ex-Soviet area (renaming and autochthonization in toponymy).
\end{abstract}

\section{MOTS-CLÉS/KEYWORDS}

degrés de traduction, nom propre, russe, transcription, translittération

Lorsqu'ils apparaissent sous telle ou telle forme dans le discours français, notamment par le biais de la traduction, les noms propres issus de textes russes (noms russes stricto sensu et ceux liés au monde russe) posent divers problèmes sur le plan pratique mais aussi sur un plan linguistique davantage théorique.

Il existe plusieurs façons de rendre les noms propres russes lors de la traduction vers des langues occidentales, notamment vers le français. La normalisation dans ce domaine reste un problème d'actualité, concernant en particulier le traitement sur Internet des noms issus des langues utilisant le cyrillique (Galenko 2002). Le passage du cyrillique à l'alphabet latin est lié à plusieurs contraintes et difficultés qui sont bien connues et décrites (cf. l'aperçu très complet dans Aslanoff 1986), mais jusqu'à présent insuffisamment systématisées. Certaines des particularités sont à étudier sur 
le plan d'une analyse proprement linguistique des noms propres (cf. Grass 2002; Superanskaja 1973).

\section{Translittération(s) ou transcription(s): Ah, ces Popoffs!}

La simplicité des faits russes est souvent trompeuse. Dans son excellent ouvrage récent consacré à la traduction du nom propre, M. Ballard (2001:27) se sert du russe, en s'appuyant sur (Dubois 1973: 498), pour illustrer la différence entre translittération et transcription: "Popov est une translittération du nom russe (elle suppose que le lecteur français sache que 'v' se prononce 'f' dans cette langue), Popoff est sa transcription.»

Cette formulation a besoin d'être nuancée. La lettre-consonne в translittérée par $v$ ne se prononce [f] que lorsqu'elle est en position faible (finale du mot ou devant une consonne sourde). Si le nom en question se met par exemple au génitif (kniga Popova «le livre de Popov»), on entend [v]. Il en est de même dans la forme féminine de ce nom de famille au nominatif: Anna Popova. Par conséquent, la notation de Попов ${ }^{1}$ par Popov recouvre une réalité importante de nature phonologique: on peut considérer cette notation comme une sorte de transcription phonologique du nom russe en question. En effet, pour Попов, la translittération Popov coïncide avec la transcription phonologique qui sera $/$ popov $^{2} /$.

D'un point de vue théorique, peut-on affirmer que la translittération Popov suppose que le lecteur français $s a c h e^{3}$ que в rendu par $v$ se prononce [f] en russe dans certaines positions? Ce serait plutôt le contraire: dans la translittération, on se place $\mathrm{du}$ point de vue de quelqu'un qui ignore ou semble ignorer ce trait du russe. Ce principe explique en partie que la forme translittérée devient ensuite, sous l'influence de l'écrit, un fait du français: il arrive en effet d'entendre des francophones prononcer les noms russes en $-o v$ avec un [v] sans l'assourdir ${ }^{4}$. Ainsi, Ivanov, le nom de l'ancien ministre russe des Affaires étrangères (en russe Игорь Иванов), était souvent prononcé [ivanov] au lieu de [ivanof] par des journalistes français, y compris par des envoyés spéciaux à Moscou.

Notons encore un détail qui complique davantage le tableau: dans certaines prononciations régionales qui remontent à un État slave ancien (Ivanov 1990: 177), le $v$ final ne s'assourdit pas, car la consonne correspondante est une sonnante bilabiale $[\mathrm{w}]$, prononcée en finale et devant consonnes, proche de $w$ en anglais. Попов se prononcera donc [popow] ou, avec altération de la voyelle non accentuée, [p $\Lambda$ pow]. Ce trait caractérise également le biélorusse où ce nom sera écrit, en suivant le principe résolument phonétique de l'orthographe biélorusse, Папоў (transcrit en français Рарой, ou, de façon plus maladroite si on omet le diacritique au-dessus de $u$, Papou).

Quant à la forme Popoff, elle correspond bien à une transcription s'il s'agit de la consonne finale de ce nom. Mais dès qu'on tient compte des voyelles, Popoff relève en partie d'une translittération: phonétiquement, dans la prononciation standard (dite «de Moscou»), la voyelle de la première syllabe n'est pas le $o$ de la seconde syllabe, car en position faible (hors accent), $o$ subit une altération et se rapproche d'un $a$ bref et fermé en devenant une voyelle centrale fermée $[\Lambda]:[\mathrm{p} \Lambda \mathrm{pof}]$ ou $[\mathrm{p} \Lambda \mathrm{po}$ : f], en marquant l'allongement de la voyelle accentuée. Une notation comme ${ }^{\star} P a p o f$ ou ${ }^{\star}$ Papoff correspondrait davantage à une transcription phonétique compte tenu de 
la prononciation standard, alors que Popoff refléterait une prononciation régionale (notamment celle des dialectes russes du Nord qui assourdissent le $v$ final).

Au regard de l'accent dynamique (appelé aussi accent tonique), la transcription courante se rapproche de la translittération: elle ne dit rien sur la place de l'accent dont le rôle en russe est considérable mais qui n'est pas noté dans l'écriture russe habituelle (exception faite d'ouvrages didactiques pour enfants et pour étrangers). On ne peut pas savoir a priori si Popov / Popoff reflète Попов (comme c'est le cas dans la prononciation russe) оu ${ }^{\star}$ Попов. La question n'est pas anodine: la variation de la place de l'accent dynamique peut avoir de l'importance. C'est ainsi que Иванов Ivanov peut être accentué à la deuxième syllabe pour faire «distingué» (Иванов) ou à la dernière syllabe pour faire "commun» (Иванов). Dans Иванов, la pièce bien connue de Anton Tchékhov ou Tchekhoff (Антон Чехов), le nom du personnage principal, qui est un universitaire aisé, se prononce bien Иванов.

Un autre fait mérite attention. Comment expliquer que l'assourdissement des consonnes russes en finale ${ }^{5}$ ne se reflète dans les transcriptions françaises traditionnelles que concernant $v$, y compris dans la toponymie (cf. Malakoff ${ }^{6}$ ) ? En effet, les toponymes comme Novgorod ou Volgograd ne sont connus des francophones que sous une forme qui coïncide avec leur translittération, sans tenir compte de la prononciation de $d$ en finale comme [t]. Des notations phonétiques telles que ${ }^{\star}$ Novgorot, ${ }^{\star}$ Volgograt (ou ${ }^{\star}$ Novgarat, ${ }^{\star}$ Valgagrat, si on veut refléter l'altération du $o$ non accentué) ne sont pas attestées.

Cela tient peut-être à la place particulière qu'occupe le couple /v/ - /f/ dans le système des consonnes russes. En effet, la lettre $\phi$ et la consonne correspondante /f/ sont historiquement marquées: à l'origine, le slave ne connaissait pas /f/ (Ivanov 1990 : 79). À l'époque du vieux russe, /f/ n'apparaissait que dans les emprunts savants au grec, au latin et au germanique, et il pouvait être remplacé dans la prononciation populaire par /p/, /x/ ou /xv/. L'existence d'un /f/ issu de l'assourdissement de /v/ est relativement récente (pas avant le $\mathrm{XIII}^{\mathrm{e}}$ siècle). Jusqu'à présent, la lettre $\phi$ qui visualise cette consonne confère au mot graphique un caractère allogène, souvent perçu comme «occidental»: aucun mot russe d'origine proprement slave ne comporte la lettre $\phi$, à quelques rares exceptions près (hypercorrections ou onomatopées). Par conséquent, on peut penser que les Russes eux-mêmes percevaient les formes en - $f(f)$ / - ef( $f)$ comme «occidentalisées», partant plus prestigieuses, et ils tendaient à les privilégier, d'autant plus qu'elles rappelaient les noms allemands en -of(f), de type Ralloff, Markloff, Echterhoff, Lullof (relevés sur <www.durben-web.de/gedcom/ surnames $>$ ).

Le doublement de $f$ peut s'expliquer aussi par le souci de rendre la lettre muette «jer»(ъ) à la fin de ces noms dans l'ancienne orthographe: Поповъ?

Un autre facteur a pu jouer: cette graphie servait sans doute à se démarquer des noms allemands d'origine slave en -ow, de type Bülow, Modrow (où $w$ ne se prononce pas).

\section{Un Иванов peut se déguiser en Иванофф}

La graphie en -of / -off (-ef ou -eff hors accent) est due à la tradition des XVIII ${ }^{\mathrm{e}}$ et $\mathrm{XIX}^{\mathrm{e}}$ siècles. Elle semble provenir de la transcription pratiquée par les Allemands; en 1814, le général comte Михаил Воронцов écrivait son nom en France: Woronzoff 
(pas Vorontsov ni Vorontsof) ${ }^{8}$. L'écrivain Иван Тургенев (mort à Bougival en 1883) orthographiait son nom Ivan Tourguéneff (cf. Tourgueniev, forme actuelle). Cette graphie a été maintenue par l'état civil allemand et français (Aslanoff 1986: 31), et elle est propre à la première émigration russe 9 .

D'où son caractère connoté: en France, le nom Ivanoff sera souvent ressenti, d'une part, comme davantage «noble» et, d'autre part, comme plus intégré au contexte français (on pense à un Français descendant de la prestigieuse émigration «blanche») par rapport à Ivanov, forme évoquant un Russe issu de l'émigration plus récente ou venant de la Russie actuelle. La différence peut être soulignée par la forme du prénom (francisé ou pas), ainsi que par l'absence de féminisation ou la féminisation du nom de famille en suivant l'usage russe. Les annuaires de France Télécom peuvent mentionner une Hélène Ivanoff à côté d'une Elena Ivanova: la distance connotative qui sépare les deux formes est patente. Voici la fréquence des différentes formes de ce nom sur <fr.yahoo.com>:

Ivanov (16 200), Ivanoff (1190), Iwanow (85), Ivanof (72), Iwanov (1), Iwanof (1). Les graphies en $w$ sont dues au passage du nom par l'allemand. Cf. pour les formes féminisées: Ivanova (2120), Iwanowa (7), Iwanova (1). Ce nom de famille est parmi les plus répandus en Russie: selon un dicton récent, dû à l'écrivain Konstantin Simonov (Константин Симонов), На Ивановых Россия держится (La Russie s'appuie sur les Ivanov).

S’il s'agit d'un Russe de la Russie actuelle, la graphie en -off ou -of paraît aujourd'hui résolument vieillie: les résultats de notre recherche sur <fr.yahoo.com $>$ montrent que le ministre russe Игорь Иванов est mentionné comme Igor Ivanov (ou Igor S. Ivanov, avec l'initiale de son patronyme, cf. à propos du patronyme Chicouène, Sakhno 2002: 213-214) dans 3420 documents, alors que la graphie Igor Ivanof n'a donné que 2 résultats. Aucun résultat n'a été obtenu pour la variante en -off: Igor Ivanoff.

Les Russes d'aujourd'hui considèrent parfois les noms d'origine russe en -of(f) $/-e f(f)$ comme autonomes, distincts de leurs équivalents en -ov / - ev. Si un Occidental se nomme Ivanoff, ce nom sera souvent rendu par Иванофф lors du passage au cyrillique. Le moteur de recherche russe <rambler.ru> recense les cas suivants: Иванофф (645), Иваноф (129), Попофф (97), Попоф (7). Certains contextes sont manifestement liés au souci (réel ou feint) de donner au nom une apparence plus imposante. Ainsi, une SARL domiciliée à Moscou, dont le directeur s'appelle sans doute Иванов, se nomme fièrement Иваноффб.

Popov / Popoff ne sont donc pas des formes absolument équivalentes sur le plan sémantique.

\section{Normes et variantes}

La translittération des caractères cyrilliques en caractères latins est réglementée par la norme française homologuée NF ISO 9: 1995 (cf. AFNOR 2000: 269-285) qui reproduit intégralement la norme internationale ISO 9: 1995. Ce système respecte les principes de translittération rigoureuse et réversible; il est particulièrement destiné au domaine de l'information bibliographique, car il fait appel au jeu de caractères codés de la norme ISO 5426. Il a cependant du mal à s'implanter, dans la mesure où il est concurrencé par la translittération dite "des slavistes", proche de la norme 
ISO/R 9: 1968, qui est largement utilisée par les russisants français (Aslanoff 1986: 40-42). Cette dernière est moins rigoureuse: ainsi, les caractères cyrilliques щ, ю et я sont rendus par des combinaisons de deux caractères: respectivement $\check{s} \check{c}$, ju et ja (cf. en ISO 9: 1995: $\hat{s}, \hat{u}, \hat{a}$ ). En annexe, nous donnons le tableau comparatif des deux systèmes.

Certes, il ne faut pas confondre translittération normalisée et translittération approximative aboutissant à une déformation phonétique du nom: selon un autre exemple cité par M. Ballard (ibid.), un Slave nommé Čurčin risque de ne pas reconnaître son nom si un francophone l'appelle en partant d'une graphie simplifiée qui oublie les diacritiques sur $c$ (cf. Curcin lu à la française). La perception par les locuteurs du nom transcrit ou translittéré est une vaste question qui doit être traitée à part: il existe une grande variété de situations qui va de la parfaite maîtrise du code par un utilisateur averti jusqu'à la méconnaissance totale du code. Voici un cas extrême du dernier type: un jour, l'auteur de ces lignes a eu la surprise de s'entendre appeler Monsieur Kakso! par un francophone ayant vu son nom écrit en majuscules cyrilliques dans sa graphie d'origine: CAXHO. La coïncidence graphique (mais non phonétique pour $\mathrm{C}, \mathrm{X}, \mathrm{H}$ ) entre caractères cyrilliques et caractères latins a permis au locuteur de traiter cette notation comme relevant du code qui lui était familier, et il l'a lue comme [kakso]. On peut remarquer que la translittération normalisée Saxno n'exclut pas une prononciation erronée [saksno] par un francophone ne connaissant pas ce système, et que la conversion courante Sakhno ne garantit pas la bonne prononciation de la consonne vélaire /x/.

Dans la pratique, on continue à faire appel à différents systèmes de transcription empirique (conversion courante) qui tiennent plus ou moins compte des contraintes orthographiques et phonétiques du français (Aslanoff 1986: 31-36). Ainsi, le nom Хрущёв (translittéré Xruščëv ou Xruŝëv) est couramment transcrit par Khrouchtchev, même si une notation de type ${ }^{\star} K h$ rouchiof rendrait mieux compte de la prononciation réelle de ce nom. À ce propos, notons que la transcription de щ par chtch (ch+ $t c h$ ) reflète une prononciation régionale et / ou vieillie [ššc'] de cette consonne, dont l'exact statut phonologique pose d'ailleurs un problème (cf. Garde 1980: 39, mais Comtet 2002:36).

Les notations habituelles comme Khrouchtchev, Gorbatchev mélangent transcription empirique et translittération: sans parler du $v$ final, soulignons que la dernière voyelle en russe n'est pas /e/ mais /o/. Ce phénomène (appelé parfois yokanié) est assez répandu en russe. Par exemple, le substantif neutre село /s'elo/ «village» voit au pluriel son accent se déplacer de la désinence au radical. Du coup, E sous accent après une consonne molle (ou une ancienne molle, comme c'est le cas des chuintantes) et devant une consonne dure devient Ё /o/: сё̈ra /s'ola/.

Il s'agit des traces d'une loi phonétique ancienne (qui a agi du XIII ${ }^{\mathrm{e}}$ au Xv $\mathrm{Xv}^{\mathrm{e}}$ s.) : la voyelle /e/ placée après une consonne molle et devant une consonne dure se transformait sous accent en /o/, car elle subissait l'effet «durcissant» de la consonne suivante. En synchronie, le phénomène se présente de façon inversée: aujourd'hui, on a le sentiment que la voyelle accentuée /o/ désignée par Ë se change en voyelle /e/ notée par E dès qu'elle se trouve en position faible (hors accent). En effet, la description phonologique du russe suggère pour le nom Дягилев (fr. Diaguileff, Diaghileff, Diaguilev) une transcription phonologique /d'ag'il'ov/, alors que phonétiquement c'est [d'ag'bl'sf], avec un $e$ non accentué altéré (se rapprochant de $i^{10}$ ). 
Le problème est que dans la grande majorité des textes russes, le tréma sur le $e$ n'apparaît pas: on écrit très souvent Горбачев au lieu de Горбачёв, d'où la notation courante Gorbatchev face à une variante bien plus rare Gorbatchov - en synchronie, cette dernière relève au niveau de la voyelle finale à la fois de la transcription phonologique et de de la transcription phonétique.

\section{Merci, Madame Gorbatchoff}

Nous avons choisi de présenter ici les différentes variantes du nom du premier président soviétique trouvées sur <yahoo.fr> (sites en français uniquement), en y ajoutant les résultats concernant deux autres noms russes non moins connus. Rappelons que Борис Ельцин (Boris El'cin) commença sa carrière politique à Екатеринбург (Ekaterinburg), grande ville dans l'Oural fondée en 1723 et nommée en l'honneur de sainte Catherine (Екатерина), l'homonyme et protectrice de l'impératrice russe Catherine Ire (cette ville s'appela Свердловск (Sverdlovsk) entre 1924 et 1991):

$\begin{array}{llll} & \text { translittération } & \text { transcript. phonologique } & \text { transcript. phonétique } \\ \text { Горбачёв } & \text { Gorbačëv } & \text { /gorbač'ov/ } & \text { [gъrb } \Lambda \text { čo:f] } \\ \text { Ельцин } & \text { El'cin } & \text { /jel'cin/ } & \text { [je:l'cyn] } \\ \text { Екатеринбург } & \text { Ekaterinburg } & \text { /jekat'er'inburg/ } & \text { [jbkъt'br'bnbu:rk] }\end{array}$

Nous proposons un essai de classification provisoire qui tient compte des divers cas mixtes ou intermédiaires. Les exemples sont classés du point de vue de leur proximité de tel ou tel type. Entre parenthèses: le nombre des documents où la forme est attestée.

1. Translittération a) exacte ou b) approximative (avec omission des diacritiques):

a) Ekaterinburg (1800); El'cin (2);

b) Gorbacev (8), cf. la translittération normale Gorbačëv; cette forme est d'ailleurs conforme aux normes orthographiques de l'italien pour la notation de/č/, mais l'italien transcrit Gorbaciov; Elcin (1).

2. Translittération + transcription phonétique:

Gorbatchev (9040); Gorbaçov (2);

Eltsin (77); Eltsine (10 500);

Ekaterinbourg (1840).

3. Transcription française quasi phonétique + translittération:

Gorbatchov (162); Gorbatchiov (1); Gorbatchef (16); Gorbatcheff (4);

Eltsyn (1); Eltsyne (1).

4. Transcription française quasi phonétique rendant plus ou moins la prononciation:

Gorbatchof (5): ex.: vodka Gorbatchof;

(L'exemple Gorbatchoff (1) est à part, car il s'agit d'une forme créée ad hoc, attestée dans la réaction d'une spectatrice ravie sortant du spectacle d'Anne Roumanoff: Merci, Madame Gorbatchoff!)

Ieltsin (7); Ieltsine (49); Yeltsin (562); Yeltsine (199);

Iekaterinbourg (340); Iekaterinburg (16); Iékaterinbourg (1); Iékaterinburg (1);

Yekaterinburg (448); Yekaterinbourg (15): les variantes en Ye- sont sans doute suggérées par l'anglais. 
5. Transcription suivant les normes d'autres langues que le français: angl.: Gorbachev (1430); Gorbacheff (1); Gorbachef (1); Gorbachov (43); Gorbachof (3);

all.: Gorbatchew (4); Gorbatchow (4); Gorbatschev (304); Gorbatschov (10); Gorbatschof (1); Gorbatschow (70, y compris Wodka Gorbatschow); Gorbatschew (3); all. et / ou néerl. : Jelzin (7); Jeltsin (3), Jeltsine (2); ital.: Gorbaciov (16); angl.: Ekaterinburgh (1): influencé sans doute par les toponymes de type Edinburgh.

6. Transcription française aberrante (métathèse de la combinaison ts rendant l'affriquée russe):

Elstine (466), Elstin (7), Yelstin (12), Yelstine (9), Ielstine (1).

7. Transcription + translittération + quasi-traduction:

Ekatherinbourg (2), Katherinburg (1) - suggérés sans doute par les équivalents anglais, allemand ou français du prénom russe Ekaterina: Catharine, Katharina, Catherine

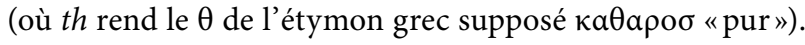

Les variantes les plus fréquentes sont observées là où le nom russe résiste le moins aux habitudes phonétiques et orthographiques du français. Les graphies en -bourg pour Ekaterinburg s'expliquent par l'analogie avec le mot bourg et les noms connus tels que Strasbourg. Ladaptation phonétique est alors probable (un francophone peut prononcer Ekaterinbourg sans $g$ ou $k$ final). Les graphies aberrantes de type Elstine (métathèse) sont dues à la rareté de la combinaison $t$ (surtout après une sonnante comme $l$ ) en français face à la fréquence de $s t$.

Pour Ельцин, il n'y a que deux tentatives de rendre la spécificité de la variante postérieure du phonème russe /i/ après une consonne dure, phonétiquement $[\mathrm{y}]$, à cause du caractère inhabituel de cette voyelle russe pour un francophone.

La notation phonétique du $E$ initial en russe comme Ie / Ié / Ye, correspondant à /je/, n'est pas rare, ce qui répond aux pratiques lexicographiques actuelles (ainsi, Le Petit Larousse 1999 fait apparaître Eltsine sans gloser et renvoie à Ieltsine). Mais elle est loin derrière la notation de type translittération (par E). D’ailleurs (Aslanoff 1986: 48) préconise Ekaterinbourg au lieu de Iékatérinbourg.

En effet, cette particularité du système alphabétique russe, qui applique en partie le principe syllabique, est difficile pour un étranger: il existe deux séries de lettresvoyelles ( $(\mathrm{a}, \ni, 0, y, ы$ / я, e, ё, ю, и), les lettres de la deuxième série indiquant a) la palatalisation de la consonne précédente, $\mathrm{b}$ ) notant, au début du mot ou après une voyelle, la combinaison consonne /j/ + voyelle (cf. Chicouène, Sakhno 2002: 43).

L'assourdissement du $g$ final dans Ekaterinburg n'est jamais rendu, à la différence du phénomène analogue concernant le $v$ dans Gorbačëv. L'altération des voyelles /e/, /o/ en position faible n'est jamais attestée. Il n'y a sur Internet rien qui ressemble p. ex. à ${ }^{\star} I$ katirinbourk, notation qui rendrait assez bien la prononciation réelle.

\section{Noms de sites web russes: quasi-translittérations jouant sur la similitude entre caractères cyrilliques et caractères latins}

Un exemple intéressant est fourni par le nom d'un site russe (d'inspiration nationaliste, détail significatif) sur Internet, que nous reproduisons en police Arial, car le graphisme est important: <http://pycckuu-gyx.ru>. 
À première vue, ce nom (pycckuu-gyx), difficilement prononçable, paraît bien étrange si on le traite du point de vue de l'alphabet latin. En réalité, il s'agit d'un jeu graphique. Les auteurs du site ont réussi à rendre la locution russe русский дух (en translittéré: russkij $d u x$ ), ce qui signifie «esprit russe, caractère russe, russité» en utilisant les lettres minuscules latines qui rappellent du point de vue graphique certaines lettres cyrilliques tout en ayant une valeur phonétique distincte. La ressemblance est certes moins évidente entre le cyrillique д (en translittéré: $d$ ) et le latin $g$, mais il faut savoir que la forme manuscrite du д coïncide avec la variante manuscrite $\mathrm{du} g$, variante proche du caractère latin en police Arial (g) ou en Courier (g). Une autre simplification est liée au fait que la lettre cyrillique й (phonétiquement [j], yod) est privée de son diacritique qui le distingue du caractère и /i/, de forme proche.

Voici un autre exemple de jeu alphabétique dans le nom d'un site russe sur Internet: <www.ycc.ru>. Il s'agit de Екатеринбургская сотовая связь «Téléphonie mobile de Ekaterinburg». La notation ycc apparaît, d'une part, comme l'abréviation d'une traduction anglaise possible du nom de cette société, en admettant que Екатеринбург soit rendu par Yekaterinburg: Yekaterinburg cellular connection. Mais, d'autre part, le sigle $c c$ peut être traité comme relevant du code graphique du cyrillique, et il correspond exactement à la locution russe сотовая связь «télécommunication cellulaire». Notons qu'un sigle comme ecc serait plus cohérent du point de vue de la graphie cyrillique du nom de Екатеринбург et de sa translittération Ekaterinburg.

\section{Degrés de traduction}

Certains cas ne sont pas clairement identifiables: ainsi, Невский проспект, nom de la célèbre avenue qui traverse le centre de Saint-Pétersbourg, est rendu normalement en français par (la) perspective Nevski / Perspective Nevski ${ }^{12}$. Conformément à l'usage russe (omission possible du mot prospekt, subst. masculin), la perspective Nevski voisine dans la traduction (Dostoïevski, p. 13) avec le Nevski (article masculin).

S'agit-il, concernant prospekt - la perspective, d'une vraie traduction, d'un calque ou d'une transcription-adaptation du mot russe? Le lien est basé sur un rapport de paronymie sur le plan étymologique (lat. pro-spect $\sim$ per-spect-). Notons que jusqu'au début $\mathrm{du} \mathrm{XIx}^{\mathrm{e}}$ s., le russe avait utilisé une forme respektiva (<perspektiva $<$ fr. perspective).

Les dictionnaires français donnent pour le mot perspective le sens d' "avenue», tout en précisant que c'est un calque du russe prospekt et en faisant comprendre que cet emploi particulier ne s'applique qu'aux réalités russes. En français, le mot perspective n'a pas ce type d'emploi: quand on parle de la perspective des Champs Élysées, on pense à la perspective créée par l'avenue des Champs Élysées. Généralement, la traduction de la toponymie urbaine russe évitera perspective en lui préférant avenue: cf. prospekt Vernadskogo (nom d'une grande avenue à Moscou) - avenue ( ${ }^{*}$ perspective) Vernadski ${ }^{13}$. Cela permet de dire que dans le rapport de traduction Nevski prospekt $=$ perspective Nevski, le terme perspective apparaît d'une certaine manière comme une partie du nom propre.

Un autre problème classique est lié à l'existence de l'«aura» sémantique de certains noms propres, dont il est difficile, voire impossible, de rendre compte dans la traduction, concernant: 
- les prénoms russes et leurs nombreux diminutifs (Breuillard 2004);

- les noms de famille "parlants» (connotés);

- les patronymes et leurs variantes.

Les observations que nous avons faites à partir de l'analyse de quelques textes littéraires russes et de leurs traductions en français montrent que, très souvent, le contenu sémantique du nom propre russe se perd en partie dans le texte français.

Chaque cas pose des problèmes spécifiques: par exemple, les différences d'emploi entre la forme officielle du prénom (celle qui est acceptée par l'état civil) et toutes les formes diminutives, ainsi que les particularités de chaque forme diminutive (liée à une certaine fonction dans le code social) sont subtiles. Ainsi, un Александр (Alexandre) sera appelé par son prénom «plein» par son chef bien plus âgé que lui (qui, selon les circonstances, peut y ajouter le patronyme), Саша (Sacha) (forme de style neutre venant de Алексаша, forme désuète) par sa femme, Сашенька (Sachenka) par sa mère aimante, Сашуля Sachoulia par sa grand-mère, Саня (Sania) оu Санька (Sanka) par un ami d'enfance, Шypa (Choura) (troncation de Caшypa [Sachoura]) par un camarade de travail. Nous transcrivons ces formes en conversion française traditionnelle, selon le principe généralement appliqué dans les traductions des textes littéraires. Ensuite, chacune des formes diminutives peut donner lieu à d'autres diminutifs plus complexes Шура $\rightarrow$ Шурик $\rightarrow$ Шурка $\rightarrow$ Шурочка; Саня $\rightarrow$ Санечка. On mesure la difficulté de la tâche liée à la traduction.

Dans les traductions, les diminutifs de prénoms russes sont parfois rendus tels quels, par transcription courante (coïncidant parfois avec la translittération). Les formes diminutives coexistent dans le texte d'arrivée avec les formes de style officiel, «neutre». Cf. (nous citons d'abord les formes «neutres»; les noms des écrivains sont aussi donnés en conversion courante):

Варвара - Varvara: Варя - Varia; Варенька - Varenka (Akounine);

Ирина - Irina: Ира - Ira (Erofeev);

Пётр - Piotr: Петрушка - Pétrouchka; Петруша - Pétroucha (Dostoїevski).

Un lecteur de la traduction française (d'ailleurs excellente) du Double de F. Dostoïevski aura du mal à comprendre pourquoi le personnage (Goliadkine) s'adresse à son domestique (mentionné comme Pétrouchka par l'auteur) en utilisant trois formes différentes du prénom, l'appelant tantôt Piotr, tantôt Pétrouchka, tantôt Pétroucha:

«- Non, non, Piotr! Non, Pétroucha, (...) Tu vois bien que c'est rien» (p. 166).

Le lecteur peut-il saisir la différence entre Piotr, forme officielle qui peut marquer la respect ou la distance, et Pétroucha, forme familière et affectueuse?

Un autre personnage, dont le prénom et le patronyme sont Емельян Герасимович - rendus par Emélian Guérassimovitch, apparaît souvent dans le texte comme Герасимыч - rendu par Guérassimytch:

"Au même instant, la porte (...) s'ouvrit et l'on vit entrer Gérassimytch, le vieux chambellan d'Olsoufi Ivanovitch.

- Voilà, Emélian Guérassimovitch, Monsieur veut entrer, et moi ...» (p. 45).

La nuance risque d'échapper au lecteur, et il est vrai qu'elle nécessiterait un long commentaire du traducteur sur ce type d'usage des patronymes dans le discours russe (cf. Chicouène et Sakhno 2002: 178). 
Quant au nom Голядкин, il est simplement transcrit par Goliadkine, sans que le traducteur indique au lecteur sa profonde signification. Or, ce nom est marqué car il se rattache au mot russe familier голядь goljad" "personne(s) de basse condition ». En revanche, le traducteur commente le nom de famille Bassavrioukov qui ne joue qu'un rôle épisodique: "Le nom rappelle celui de Bassavriouk, «le diable à image humaine» d'une nouvelle de Gogol, Le soir de la Saint-Jean» (p. 250). Ce commentaire semble en rapport avec le contexte immédiat, car Goliadkine, entendant ce nom annoncé par un laquais, pense: «Une famille de bonne noblesse, originaire d'Ukraine. » Certes, le commentaire du traducteur n'indique pas au lecteur (qui peut ne pas le savoir) que la nouvelle de N. Gogol (Гоголь) se situe en Ukraine et que Gogol lui-même était d'origine ukrainienne.

Outre la perspective Nevski, les autres toponymes célèbres de Saint-Pétersbourg sont transcrits avec une note du traducteur, p. ex. pour Litejnyj (prospekt): le Litéïny (expliqué comme « une des principales rues perpendiculaires à la perspective Nevski », p. 14), même si le nom de cette avenue se traduit comme «avenue des Fondeurs / des Fonderies». En revanche, le nom, bien moins connu, de la rue où habite le personnage principal, Goliadkine, n'est pas transcrit comme rue Chestilavotchnaïa (lourd et opaque pour le lecteur) mais traduit: rue des Six-Boutiques (p. 8), ce qui ajoute du pittoresque à la description du triste logement mal entretenu du héros.

Les exemples de traduction des zoonymes ou anthroponymes "parlants» sont assez rares. Dans la nouvelle de M. Boulgakov Cour de chien, le personnage central s'appelle Шарик Šarik; il s'agit d'un chien errant qui se transforme en être humain à la suite d'une opération chirurgicale, et son nom devient un nom de famille ordinaire en -ov: Шариков. Le traducteur rend Шарик par Boule, car ce nom signifie "(petite) boule», "(petit) ballon» et c'est un diminutif du mot шap «boule», «sphère». On ne pouvait guère faire autrement, puisque le texte comporte un commentaire ironique sur le contraste entre l'apparence du chien et le sens du nom:

«Boule, l'a-t-elle appelé... Idée saugrenue, non? Boule, c'est quelqu'un de rond, de replet et bête, qui s'empiffre de flocons d'avoine, possède un pedigree; alors qu'il n'estqu'un animal hirsute, long comme un jour sans pain et tout couturé, clébard efflanqué et sans domicile fixe.» (p. 210)

Ensuite, en toute logique, le nom de famille Шариков est traduit par Boulov. Le problème est que Шарик correspond à un nom de chien répandu en Russie et tellement banal (cf. Médor en français) qu'il en devient presque désémantisé: quand un Russe d'aujourd'hui l'entend, il ne pense guère à son sens étymologique, ce nom évoque pour lui surtout l'image d'un chien «de rue», d'apparence ordinaire, sans pedigree. Il est possible cependant qu'à l'époque décrite par Boulgakov (années 1920), la banalisation de ce nom était moins importante. N'empêche que pour un francophone, Boule est un nom très marqué, tout à fait inhabituel pour un grand chien errant qui ne ressemble pas à une boule. Par conséquent, la contradiction entre le physique de l'animal et son nom est radicalisée dans le texte français par rapport à l'original russe.

\section{Le nom du petit chien du duc d'Enghien}

La variabilité des noms propres russes est un autre problème de traduction, problème lié à : 
a) la coexistence dans le discours russe contemporain, à la suite de débaptisations et de rebaptisations successives, des différentes appellations, toutes plus ou moins connotées, d'un même objet toponymique (cf. Ballard 2001 : 143), p. ex. pour Saint-Pétersbourg: Sankt-Peterburg, Peterburg, Piter, Leningrad - formes translittérées); paradoxalement, la région administrative dont Saint-Pétersbourg est le cheflieu se nomme toujours Leningradskaja oblast' "région de Leningrad»;

b) l'«autochtonisation» des noms propres des ex-républiques soviétiques; cf. le nom de le ville biélorusse de Могилёв (orthographe russe, cf. en translittéré: Mogilëv) - fr. (conversion courante) Moguilev (<yahoo.fr>: 301), Moghilev (97) et Магілёў (orthographe biélorusse) - transcription attestée en français dans un ouvrage récent (A. Goujon, V. Simaniec, Parlons biélorussien. Paris: L'Harmattan, 1997): Mahilou, cette dernière forme présentant pour un francophone moyen une apparence vaguement tahitienne ou hawaïenne plutôt que slave.

Voici d'autres variantes trouvées sur <yahoo.fr>: Mogilev (277), Moguilov (3), Moguiliov (3), Moghiliov (1), Mohilow (5), Mohilew (14), Mohiloff (8). La dernière forme, Mohiloff, servit de nom au célèbre chien du duc d'Enghien, qui l'accompagnait pendant son exécution en 1804. Sous la plume de Catherine II, cette ville avait été mentionnée comme Mohilow ou Mogilof (Davidenkoff 1997: 255, 259). En revanche, Mahileu (1) est récent.

Un autre exemple est constitué par le nom russe de la capitale moldave Кишинёв (translittéré du russe Kišinëv): Kisinev (<yahoo.fr>: 7), Kichinev (471), Kichiniov (3), Kichinov (2), Kichineff (15), Kichineff (9), Kishinev (477), Kishinov (2), Kishiniov (2); en moldave cyrillique Кишинэу, d'où Kichineu (1), Kishineu (1), mais moldave moderne (utilisant l'alphabet latin version roumaine): Chişinau, d'où Chisinau (5240), Chishinau (5), ainsi que des formes hybrides Kichinau (13) et Chisineu (2 - à ne pas confondre avec Chisineu Cris, ville en Roumanie).

La variété de formes est impressionnante, tout autant que la distance entre Kichineff et Chisinau (qu'un francophone risque de lire [šizino]).

\section{Conclusion}

Ainsi, au-delà de la trompeuse simplicité des «règles» de transcription et de translittération et en dépit de son apparente banalité («il sert à désigner / identifier telle personne, ou tel lieu, etc.»), le nom propre russe pose de multiples problèmes de traduction et nous donne souvent l'occasion de réfléchir sur le système linguistique du russe et sur le statut du nom propre en général.

\section{NOTES}

1. Ce nom de famille (signifiant «fils / descendant du pope»), répandu parmi les Russes, est devenu en français une sorte de surnom désignant les Russes en général (Ah, ces Popovs / ces Popoffs!).

2. Nous indiquons l'accent tonique en mettant en gras la lettre-voyelle accentuée. Les transcriptions phonologiques sont signalées par / /, alors que les transcriptions phonétiques sont mises entre [ ].

3. Cette idée est d'ailleurs absente de (Dubois 1973: 498) où le principe de translittération est formulé comme ceci: «se contenter de rechercher, pour chaque lettre ou suite de lettres, une lettre ou une suite de lettres sans s'inquiéter des sons effectivement prononcés». Selon Garde (1980: 46), «la translittération [...] ne suppose aucune information particulière sur sa prononciation [celle du mot]».

4. Cela concerne même certains russophones cultivés lorsqu'ils parlent français: ils croient bien faire en suivant la prononciation des noms français de type Debove, Vanhove, où ils essayent de ne pas assourdir la consonne finale. 
5. L'assourdissement de $v$ au milieu d'un mot n'est jamais montré dans les transcriptions: le nom du célèbre compositeur Чайковский est connu en français comme Tchaïkovski (parmi d'autres variantes), mais jamais avec un $f:{ }^{\star}$ Tchaïkofski. Cependant, le poète В. Тредиаковский signait en français son nom: Trediakoffski (précision que nous devons au Professeur J. Breuillard, Université Paris IV).

6. Précisons que Malakhof(f) serait une notation plus exacte. Il s'agit de la célèbre colline (en russe Малахов курган), point central de la défense de Sébastopol, prise d'assaut en 1855 par la division de Mac-Mahon. Quant à la ville de Malakoff, son nom perpétue le souvenir du café À la tour de Malakoff, dont le patron avait fait la guerre de Crimée.

7. Nous devons cette hypothèse au professeur Roger Comtet (Université Toulouse II).

8. Nous remercions le Professeur Jean Breuillard (Université Paris IV) de nous avoir fourni ces indications.

9. Les toponymes et les noms de famille bretons en -off ont certes une origine distincte, cf. Roscoff (< celte ros «butte» + goff «forgeron»).

10. Nous symbolisons par [y], une voyelle proche d'un $i$ postérieur (« $i$ dur»), par [b]: un [i] bref et ouvert prononcé en pensant à /e/ fermé; par [ъ] : une voyelle centrale proche du «e muet» français non arrondi; par $[\Lambda]$, une voyelle centrale proche de /a/ fermé. Le signe ' marque les consonnes palatalisées (molles).

11. Elle pose parfois problème, et il faut dans certains cas la prouver par la mise de la voyelle en position forte, sous accent, en faisant appel aux formes dont ces noms sont issus. C'est possible pour /o/ et /a/ de Горбачёв qui vient du surnom Горбач, «Le Bossu», issu du substantif russe горб «bosse». Mais c'est plus difficile p. ex. pour /e/ de Екатеринбург lié au prénom Екатерина: on ne peut l'établir que lorsqu'on connaît la forme grecque du nom de sainte Catherine d'Alexandrie

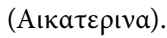

12. C’est par ailleurs le titre de la célèbre nouvelle de Nicolas Gogol écrite en 1835 .

13. Notons que le nom de famille est au génitif dans la construction russe (mot à mot: «avenue de Vernadski»). La possibilité de rendre ce génitif dans la traduction est une question à part qui ne sera pas abordée ici.

14. Variantes: $k h, h$.

15. Variante: $\dot{e}$.

\section{RÉFÉRENCES}

AFNOR (Association française de normalisation) (2000): Documentation, T.1: Présentation des publications et recherche documentaire, Paris, AFNOR.

Aslanoff, S. (1986): Manuel typographique du russiste, Paris, Institut d'études slaves.

BALlARD, M. (2001): Le nom propre en traduction: anglais - français, Paris, Ophrys.

Breuillard J. (2004), "Diminutifs des prénoms russes», - SLOVO, vol. 30-31, p. 177-209.

Chicoul̀ne, M. et S. SAKhno (2002): Parlons russe: Une nouvelle approche, Paris, L'Harmattan.

Cомтет, R. (2002) : Grammaire du russe contemporain, Toulouse, PUM.

Davidenkoff, A. (dir.) (1997): Catherine II et l'Europe, Paris, Institut d'études slaves.

Dubois, J. et al. (1973): Dictionnaire de linguistique, Paris, Larousse,.

Galenko, V. T. (2002): «Kommunikacionnoe alfavitnoe pis'mo nelatinskix jazykov», Rusistika segodnja 1-4, p. 239-248.

Garde, P. (1980): Grammaire russe: Phonologie. Morphologie, Paris, Institut d'études slaves,.

Grass, TH. (2002): "La traduction des noms propres allemands: approche conceptuelle et approche syntaxique», Journée d'études La traduction des noms propres, Université Paris $\mathrm{X}, 6$ avril 2002.

Ivanov, V. V. (1990): Istoričeskaja grammatika russkogo jazyka, Moskva.

Superanskaja, A.V. (1973) : Obščaja teorija imeni sobstvennogo, Moskva, Nauka.

\section{Textes analysés et leurs traductions}

Arounine, B. (2001): Tureckij gambit, Moskva, Zaxarov. - Le Gambit turc, Paris, Pr. de la Cité (trad. par I. Sokologorsky).

Boulgakov, M. (2000-1990): Sobače serdce. Moskva: OLMO. - Coeur de chien, Moscou, Radouga (trad. par C. Staïanov et A. Karvovski). 
Dostoïevski, F. (1998): Dvojnik - Le Double, Leméac, Actes Sud (trad. par A. Markowicz). Erofeev, V. (1990): Russkaja krasavica. - La Belle de Moscou, Paris, Albin Michel (trad. par A. Pingaud, L. Jurgenson).

\section{ANNEXE}

\section{Caractère cyrillique}

Aa

Б6

$\mathrm{BB}$

Гг

Дд

$\mathrm{Ee}$

Ëë

Жж

33

Ии

Йй

travail

Кк

Лл

$\mathrm{Mm}$

$\mathrm{HH}$

Oo

Пп

$\mathrm{Pp}$

$\mathrm{Cc}$

TT

yy

$\Phi \phi$

$\mathrm{Xx}$

Цц

Чч

Шш

Щщ

Ђъ

Ыы

bь

Ээ

Юю

Яя
ISO/R 9:1968

translittération

\section{Aa}

$B b$

Vv

$G g$

Dd

Ee

$\ddot{E} \ddot{e}$

$\check{Z} \check{z}$

$Z z$

Ii

Jj

Kk

Ll

$\mathrm{Mm}$

$\mathrm{Nn}$

$\mathrm{Oo}$

$P p$

$R r$

Ss

$T t$

Uu

Ff

$X x^{14}$

Cc

$\check{C} \check{c}$

$\check{S} \check{s}$

ŠČšč

" (signe dur)

$Y y$ (i dur)

' (signe mou)

$\grave{E} \grave{e}^{15}$

JUju

Jaja
ISO 9:1995

Aa

$\mathrm{Bb}$

Vv

Gg

Dd

Ee

Ëë

$\check{Z} \check{z}$

$\mathrm{Zz}$

Ii

$\mathrm{Jj}$

$\mathrm{Kk}$

$\mathrm{Ll}$

$\mathrm{Mm}$

$\mathrm{Nn}$

Oo

$\mathrm{Pp}$

$R r$

Ss

$\mathrm{Tt}$

$\mathrm{Uu}$

Ff

$\mathrm{Hh}$

$\mathrm{Cc}$

Čč

Šš

$\hat{S} \hat{s}$

"

Yy

Èè

Ûu

Ââ transcription
phonologique

/a/

/b/ ou /b'/

$/ \mathrm{v} /$

/g/ ou /g'/

/d/ ou /d'/

/'e/, /je/ ou /e/

/'o/; /jo/

/ž/ ou /š/

$\mid z /$ ou $/ z^{\prime} \mid$

/i/, /'i/

$/ \mathrm{j} /$

$/ \mathrm{k} / \mathrm{ou} / \mathrm{k}^{\prime} /$

/l/ ou /l'/

$/ \mathrm{m} /$ ou $/ \mathrm{m}^{\prime} /$

$/ \mathrm{n} /$ ou $/ \mathrm{n}^{\prime} /$

/o/

$/ \mathrm{p} /$ ou $/ \mathrm{p}^{\prime} /$

$/ \mathrm{r} /$ ou $/ \mathrm{r}^{\prime} /$

/s/ ou /s'/

$/ \mathrm{t} /$ ou $/ \mathrm{t}^{\prime} /$

$/ \mathrm{u} /$

/f/ ou /f'/

$/ \mathrm{x} /$ ou $/ \mathrm{x}^{\prime} /$

/ts/

$|\breve{c}|$

|šl

$|\breve{s}:|$

aucune

/i/

aucune

le/

/'u/, /ju/

$/$ a /, /ja/ valeur phonétique approximative

presque comme A français

comme B français

comme V fr. dans valse

comme $\mathrm{G}$ français dans grand

comme $\mathrm{D}$ français dans donner

comme $\mathrm{E}$ français dans l'été

ou YE dans yé-yé

comme $\mathrm{O}$ fr. dans national

ou IO dans iode

comme J fr. dans jambe

comme $\mathrm{Z}$ fr. dans zéro

comme I fr. dans vie

un peu comme IL fr. dans

comme $\mathrm{K}$ fr. dans karaté comme L fr. dans double ou comme dans lit comme $\mathrm{M}$ fr. dans mot comme $\mathrm{N}$ fr. dans notre comme $\mathrm{O}$ fr. dans porte comme $\mathrm{P}$ fr. dans père comme R fr. dans rat (prononcé roulé) comme S fr. dans son comme $\mathrm{T}$ fr. dans ton comme OU fr. dans outre

comme F fr. dans fort comme $\mathrm{CH}$ allemand dans nach comme TS fr. dans tsé-tsé, tsar comme TCH fr. dans match comme $\mathrm{CH}$ fr. dans châle un peu comme $\mathrm{CH}$ fr. dans Chut!

(marque la dureté de la consonne) un peu comme I angl. dans thin

(indique que la consonne est mouillée)

comme E fr. dans être

comme OU fr. dans Sioux ou comme YOU dans Yougoslave comme A fr. dans liane ou YA dans yack 